\title{
Transmission Spectra of Materials in the Terahertz Range Measured by a Hot Electron Bolometer-Based System
}

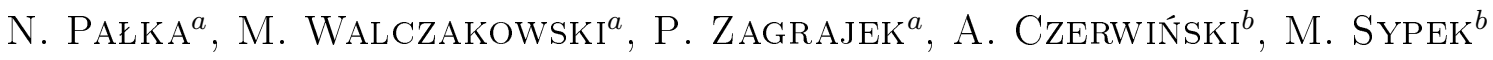 \\ AND M. SzUSTAKOWSKI ${ }^{a}$ \\ ${ }^{a}$ Military University of Technology, S. Kaliskiego 2, 00-908 Warszawa, Poland \\ ${ }^{b}$ Warsaw University of Technology, Koszykowa 75, 00-662 Warszawa, Poland
}

\begin{abstract}
This paper presents an optical system for transmission spectral measurements in the terahertz range. The source of radiation is a tunable solid-state laser, which generates a narrow-band nanosecond pulses in the range $0.67-$ $2.68 \mathrm{THz}$ and based on optical parametric oscillator phenomenon. The signal is detected with a fast and sensitive hot electron bolometer. Principle of generation and detection of pulses as well as methodology of measuring spectral characteristics are presented. We compared the results obtained with the free-space setup, which is based on the optical parametric oscillator and hot electron bolometer, with a purged time domain spectroscopy system in the range of $0.7-2.2 \mathrm{THz}$.
\end{abstract}

DOI: $10.12693 /$ APhysPolA.124.538

PACS: 07.57.Ty, 78.20.Ci, 78.40.-q, 78.47.jg

\section{Introduction}

Explosive-related threats in public transport, especially in aerial transport, critical infrastructural facilities, like railway stations, airports, undergrounded railways, and water supplies, as well as in military operations result in development of new technologies for screening people and baggage to find not only explosive materials but also guns and other dangerous materials like biological weapon, radioactive materials and drugs. The most popular sensors base on electromagnetic metal detectors, X-rays, mass spectrometry, chemiluminescence, laser techniques and others [1].

On the other hand, the $\mathrm{THz}$ technology is still one of the promising candidate for detection and identification of explosive materials. The development of terahertz techniques is carried out simultaneously in many directions. Due to the non-ionizing properties of the $\mathrm{THz}$ radiation, it can be used while dealing with living organisms or organic materials [2]. A growing number of applications in security systems allows for the gradual replacement of X-ray equipment. A feature making the terahertz radiation so attractive are characteristic absorption bands of many substances lying in the range of $0.3-10 \mathrm{THz}$. This group includes some drugs and explosives [3, 4], which makes use of the transmission and reflectance spectroscopy fastest growing within the terahertz technology. This paper illustrates the operation of the transmission spectroscopy in the $0.7-2.2 \mathrm{THz}$.

\section{Experimental setup}

We used the latest commercially available equipment to build the experimental setup capable of making progress in the field of $\mathrm{THz}$ transmission spectroscopy. The source of radiation is the solid-state laser system from MSquare [5], that bases on phenomenon of optical parametric oscillator (OPO) (Fig. 1) to generate narrow-band, nanosecond pulses in the range 0.7-2.2 THz. Pulsed operation is achieved by modulation of the cavity $Q$ factor. The laser repetition rate is $53 \mathrm{~Hz}$.

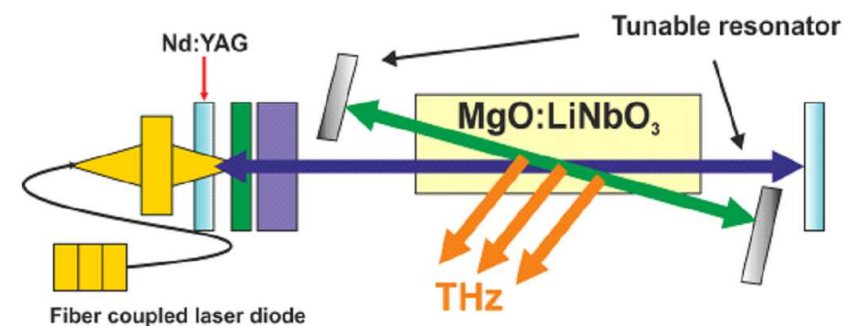

Fig. 1. The OPO optical system used to generate the terahertz pulses.

The nonlinear lithium niobate crystal doped with magnesium oxide $\left(\mathrm{MgO}: \mathrm{LiNbO}_{3}\right)$ is placed in a tunable optical resonant cavity, which also have a purpose of active region. A pumped signal, incident onto the crystal is converted into two signals with lower frequencies according to the relationship [6]:

$$
\omega_{\mathrm{p}}=\omega_{\mathrm{i}}+\omega_{\mathrm{THz}}
$$

where $\omega_{\mathrm{p}}$ is the pumping frequency, $\omega_{\mathrm{i}}$ is the idler signal, and $\omega_{\mathrm{THz}}$ is the terahertz frequency signal.

This is one case of a most general three-wave interaction in which energy and momentum are conserved. Graphical interpretation of the noncollinear phase matching is shown in Fig. 2.

The nonlinear crystal is placed in such a way that the idler and the terahertz signal can be amplified during the subsequent pass through the resonator. Partial spatial collinearity of the pumping, idler and terahertz signals in a nonlinear crystal leads to an idler and terahertz signal gain (parametric amplification) and the cor- 


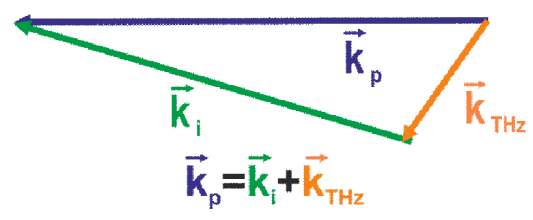

Fig. 2. Noncollinear phase matching.

responding weakening of the pump signal. Increasing the pump power above the threshold power strengthens the idler and terahertz signal with a value greater than losses caused by pass through the resonator.

Tunability of the laser is obtained by changing the conditions of the interaction pump signal with the crystal (for example, by changing the angle of the crystal, the temperature or changing the geometry of the input beam to the crystal), wherein the differential and terahertz signal are tuned simultaneously at the same time. Tuning range is limited only by absorption and reflection of the crystal inside resonator. For narrowing the spectral width of the generated signal selective optical elements are placed in the system.

The terahertz signal is detected by the hot electron bolometer (HEB) [7]. The sensing component is a $4 \mathrm{~nm}$ thick bridge made of niobium nitride $(\mathrm{NbN})$ integrated with a planar logarithmic spiral antenna on a substrate of highly resistive silicon [8]. Aperture of the detector input is limited to $1-2^{\circ}$. The terahertz signal gets to bolometer through a window made of the high density polyethylene (HDPE), afterwards beam is passing by the infrared filter limiting the bandwidth up to $0.3-3.0 \mathrm{THz}$. The filtered signal is collected by a silicon lens and focused on the bridge (Fig. 3). Due to the characteristics of electron transitions and the sensitivity of the active material, the detector operating temperature is set to $8.5 \mathrm{~K}$.

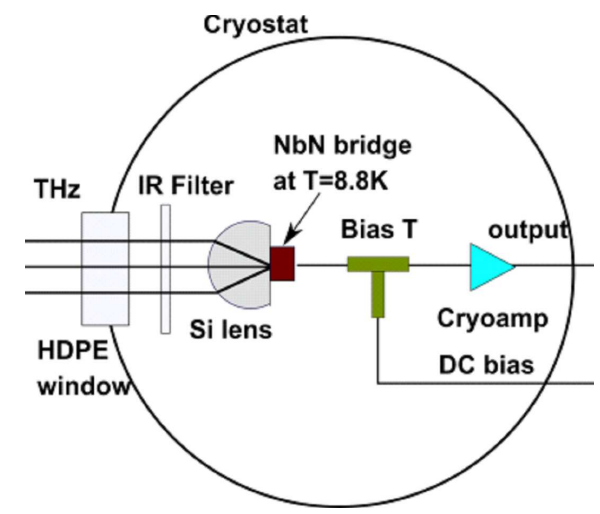

Fig. 3. Schematic diagram of the bolometer.

Figure 4 shows a diagram of the measurement system. The OPO is a source of two signals: the optical idler signal with the wavelength of about $1067 \mathrm{~nm}$ and the terahertz signal. The idler pulse is introduced into the optical fiber and after splitting by the coupler is simul- taneously measured by the spectrum analyzer (Fig. 5a) and used as a trigger for an oscilloscope. Measurement of the spectrum allows us to determine the frequency of the terahertz signal by means of Eq. (1).

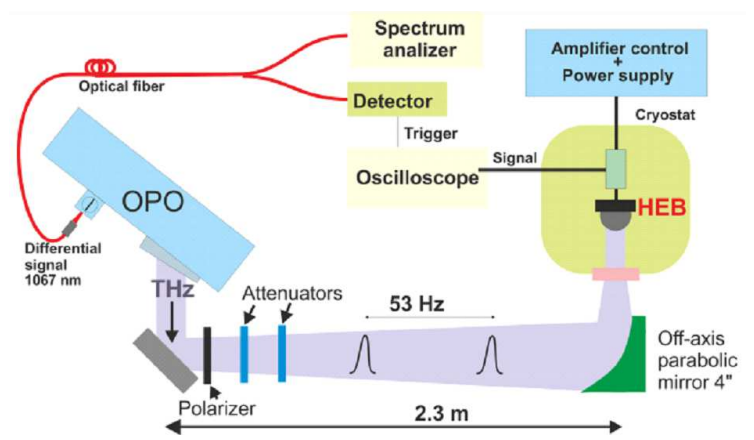

Fig. 4. The experimental setup.
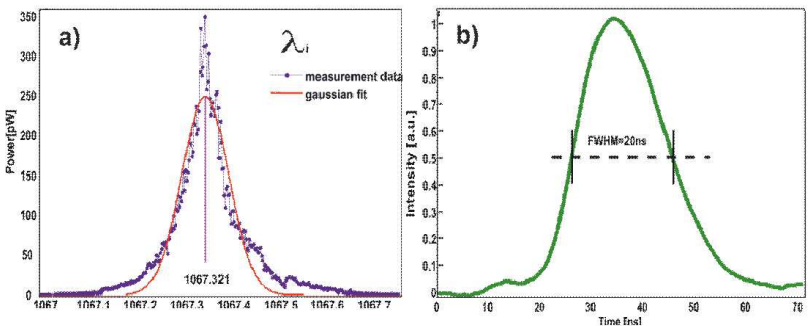

Fig. 5. The differential pulse measured by the spectrum analyzer (a) and the terahertz pulse measured at $1 \mathrm{THz}(\mathrm{b})$.

The terahertz beam first passes through a polarizer, which provides linear polarization of the beam relevant in spectroscopy of some materials. Afterwards, due to a low dynamic range of the detector, the $\mathrm{THz}$ beam is attenuated by a set of attenuators. The optical path between the source and the detector is about $2.3 \mathrm{~m}$. The optical path could be shorter, but it was extended to reveal a potential of the setup. The distance allowed to locate the bolometer and the OPO on separate optical tables and makes it possible to easy change the setup for a reflective spectroscopy configuration.

Spectral response measurement of material is carried out by tuning the laser and collecting data point by point in the frequency domain. Due to the different durations of the pulses at different frequencies, information about the spectral properties of the test material is determined on the basis of the pulse energy. The average terahertz pulse duration is about $20 \mathrm{~ns}$ (Fig. 5b), but is not constant. Taking into account the fluctuations in the shape of the pulse, changes of the optical path length that translate into changes of the position of the pulses in time, each received signal is processed based on parameters of the correspondent pulse uses for triggering the oscilloscope. The pulse energy is calculated by integrating the signal envelope in the pre-designated limit. Uncertainty 
of measurements of each point of measurement is reduced by averaging at least 30 pulses. The first step in determination of the spectral characteristic is a reference measurement - transmission through the setup without the sample. Afterwards, transmission through the sample is measured. Finally, the transmission of the sample is obtained by comparing these two results.

Sample preparation is a very important factor to obtain reliable spectra. Attenuation of most pure materials is too high to obtain reliable measurements in $\mathrm{THz}$ range, so in order to prepare samples $10 \%$ of material was mixed with $90 \%$ of polyethylene to obtain a sample with weight of $400 \mathrm{mg}$. Afterwards, the powder was loaded to a form and pressed with 2 tons of pressure. As a result, a pellet with diameter of $13 \mathrm{~mm}$ and thickness of about $3 \mathrm{~mm}$ was obtained. The samples of the following materials were prepared: salicylic acid and two explosive materials: tetranitrate (V), tetrahydroksymetylometan (PETN), and hexogen (royal demolition explosive, RDX) [9-11].

\section{Results}

Figures 6-8 present the transmission spectra of the salicylic acid, PETN and RDX, respectively. The results are presented as absorbance according to the relationship

$$
A(f)=-10 \log \left(\frac{I_{1}(f)}{I_{0}(f)}\right),
$$

where $I_{0}(f)$ is the reference measurement and $I_{1}(f)$ is the measurement of the sample.

These results were compared to spectra (Fig. 6-8) measured with a time domain spectroscopy (TDS) system with the following parameters: spectral range 0.063.6 THz, signal-to-noise better than 4000:1, dynamic range higher than 3 OD in the range $2 \mathrm{~cm}^{-1}$ to $100 \mathrm{~cm}^{-1}$, spectral resolution $0.06 \mathrm{THz}$ and rapid scan mode with $30 \mathrm{scans} / \mathrm{s}$. The TDS results were obtained in dry atmosphere with humidity less than $1 \%$.

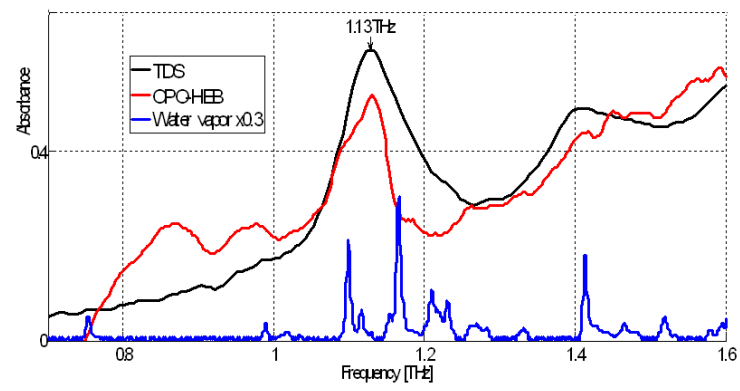

Fig. 6. THz spectra of the salicylic acid sample.

The OPO-HEB setup worked in open space at room temperature with humidity of about $23 \%$. Therefore, Figs. 6-8 also show the absorbance spectra of air with humidity of $25 \%$ measured by the TDS setup. This fact implies presence of water vapor absorption peaks in the

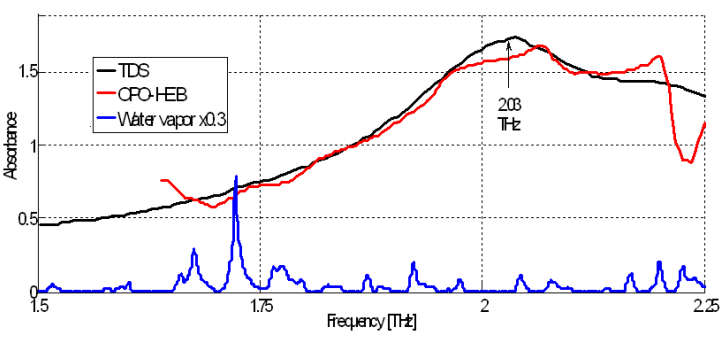

Fig. 7. THz spectra of the PETN sample.

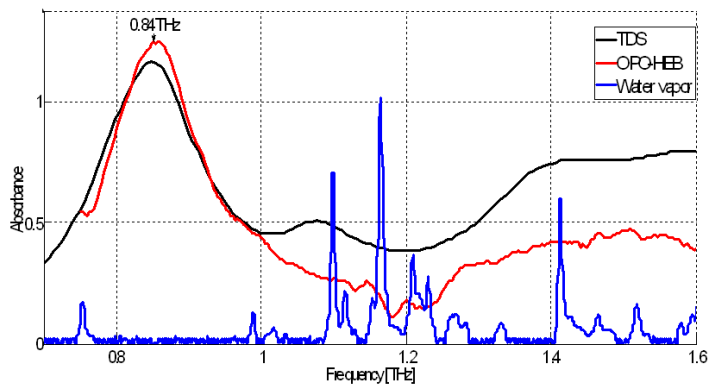

Fig. 8. THz spectra of the RDX sample.

measured characteristics. Since the material and reference transmission spectra were measured in the same conditions, so water vapor influence on the absorption spectra of materials should be eliminated. However, we can notice minor absorption features typical for water vapor in Figs. 6-8. Closer look into all three spectra shows correlation between water vapor absorption peaks and distortions in the measured material spectral characteristics. We think that the source of this effect in our experiment was associated with different configuration of the attenuators used in the reference and the material measurement. This corresponds with the difference in the optical power used for both measurements, which makes influence of water vapor noticeable.

\section{Conclusions}

In this paper we report on the terahertz absorption spectra of three materials: salicylic acid, RDX, and PETN, measured in the atmosphere with humidity of about $23 \%$. The details of the tunable OPO, the fast and sensitive bolometer as well as the measurement setup and methodology are presented.

The considered OPO-HEB setup shows good agreement of the spectral characteristics in comparison to the TDS method. The typical spectral features of the considered materials can be identified using both methods in the range $0.7-2.0 \mathrm{THz}$. However, the TDS setup seems to have better performance in terms of signal-to-noise ratio and sensitivity above $2 \mathrm{THz}$.

The TDS measurements were carried out in the dry atmosphere (humidity $<1 \%$ ) and the optical path of the $\mathrm{THz}$ beam was about $30 \mathrm{~cm}$. In case of the OPO-HEB 
system (humidity $\approx 23 \%$ ), some minor influences of the water vapor on the spectra can be observed due to the $3 \mathrm{~m} \mathrm{THz}$ beam path.

However, these influences are not too strong and taking into account the tunability and sensitivity of this system, it reveals a potential for stand-off identification of dangerous materials in the reflection configuration.

\section{Acknowledgments}

The research work was carried out in the framework of the Project of the Ministry of Science and Higher Education No. N515 020140 .

\section{References}

[1] Z. Bielecki, J. Janucki, A. Kawalec, J. Mikołajczyk, N. Palka, M. Pasternak, T. Pustelny, T. Stacewicz, Wojtas, Metrol. Meas. Syst. 19, 3 (2012).

[2] L. Dexheimer, Terahertz Spectroscopy: Principles and Applications, CRC Press, 2008.
[3] Y. Hu, P. Huang, L. Guo, X. Wang, C. Zhang, Phys. Lett. A 359, 728 (2006).

[4] K. Gut, Bull. Pol. Acad. Sci., Techn. Sci. 59, 395 (2011).

[5] Firefly-THz User Manual, MSquared Lasers Ltd., Glasgow 2011.

[6] D. Molter, M. Theuer, R. Beigang, Opt. Expr. 17, 6623 (2009).

[7] Superconducting Nanotechnology, HEB User Manual, Scontel, Moscow 2011.

[8] A.D. Semenov, G.N. Gol'tsman, J. Appl. Phys. 88, $502(2000)$.

[9] J. Chen, Y. Chen, H. Zhao, G.J. Bastiaans, X.-C. Zhang, Opt. Expr. 15, 12060 (2007).

[10] M. Zyczkowski, M. Szustakowski, P. Markowski, M. Karol, Acta Phys. Pol. A 122, 939 (2012).

[11] N. Palka, Acta Phys. Pol. A 118, 1229 (2010). 